\title{
RELACIÓN DE LOS MENORES CON LAS REDES SOCIALES Y EL WHATSAPP: CAUSAS Y CONSECUENCIAS
}

\author{
Tamara Moreno Martín \\ Educadora Social \\ tamaraedsocial@gmail.com \\ Ana Isabel Isidro de Pedro \\ Profesora Titular de E.U. \\ Departamento de Psicología Social y Antropología. \\ Universidad de Salamanca \\ anyis@usal.es
}

Fecha de Recepción: 3 Abril 2018

Fecha de Admisión: 10 Abril 2018

\section{RESUMEN}

En la actualidad, el smartphone y las Redes Sociales se han convertido en elementos casi obligados para todo el mundo, desde edades muy tempranas, constituyendo una nueva y poderosa forma de socialización, con funciones relacionales y comunicativas tan potentes que, si no utilizas alguna de ellas, parece que 'no existes'. Y éste es, precisamente, el motivo que nos ha llevado a desarrollar el presente trabajo, donde pretendemos estudiar las causas y consecuencias derivadas del tipo de relación establecida por los menores con las Redes Sociales y el WhatsApp. Se analiza la influencia de algunos factores como la autoestima y la necesidad de atención y reconocimiento social - llegando incluso al narcisismo- cristalizada en la incesante 'subida' (publicación) de fotos e información personal, así como otras consecuencias negativas que pueden derivarse de una utilización poco responsable y/o abusiva, como la dificultad para interaccionar con otros fuera de una pantalla 0 las posibilidades de control del otro que otorgan. Asimismo, se pretenden analizar los motivos que hacen tan atractivas estas herramientas y cómo afectan a la vida de los usuarios, centrándonos en diferentes aspectos del comportamiento social de los mismos. Para llevar a cabo la investigación se utilizó una metodología cuantitativa, a través de un cuestionario ad hoc, de elaboración propia, aplicado a una muestra de alumnos de la provincia de Salamanca, obteniendo una muestra final de $n=134$, con menores estudiantes de ESO, Bachillerato y FP, con media de edad de 14,8 años. Los resultados permiten concluir que estas herramientas pueden llegar a suponer una importante limitación en la vida de los usuarios, dificultado aspectos de su vida social, llegando incluso a que su estado de ánimo dependa de la retroalimentación obtenida en las redes a través de las publicaciones, no siendo tan sencillo como piensan desvincularse de ellas. 


\section{RELACIÓN DE LOS MENORES CON LAS REDES SOCIALES Y EL WHATSAPP: CAUSAS Y CONSECUENCIAS}

Palabras clave: menores; redes sociales; WhatsApp; móvil; socialización

\section{ABSTRACT}

Relationship of minors with social networks and whatsapp: causes and consequences.

At present, the smartphone and social networks have become almost mandatory elements for everyone, from very early ages, constituting a new and powerful form of socialization, with relational and communicative functions so powerful that if you do not use any of them, it seems that "you do not exist". This is the reason that led us to develop the present work, intending to study the causes and consequences derived from the type of relationship established by minors with social networks and WhatsApp. The influence of certain factors such as self-esteem and the need of attention and social recognition -even reaching narcissism- which crystallize in the incessant uploading (publication) of photos and personal information, is analysed. Moreover, other negative consequences that may result from a little responsible and/or abusive use, such as the difficulty of interacting with others outside a screen or the possibilities to control to the other that they can allow, are also considered. Likewise, by focusing on different aspects of their social behaviour, we intend to analyse the reasons that make these tools so attractive and how they affect the lives of the users. To perform this research, a quantitative methodology was used, through an ad hoc questionnaire developed by the authors. It was applied to a sample of $n=134$ students from the province of Salamanca: ESO (Obligatory Secondary Education) children, high school and professional training students, with an average age of 14.8 years. From the results obtained it is possible to conclude that these tools can represent an important limitation in the life of the users, making difficult some aspects of their social life, even doing that their mood depend on the feedback obtained from the networks through the publications, not being as simple as they think to be separated from them.

Keywords: minors; social networks; WhatsApp; mobile; socialization

\section{ANTECEDENTES}

Según señala Aguilera (2013),

El 93\% de los internautas españoles tienen una cuenta activa en al menos una red social. Por encima de los ordenadores, comienzan a imponerse los móviles Smartphone (67\% de usuarios) y las Tablets (56\%) como los dispositivos más usados. Facebook sigue siendo la red líder absoluta de las redes (83\%) seguida de Twitter (42\%), Tuenti $(27 \%)$ y Google+ $(27 \%)$. Redes sociales generalistas (Facebook, Twitter, Google+), profesionales (Linkedin), cerradas o abiertas, se van instalando en nuestra vida y las utilizamos cada vez más para relacionarnos, informarnos, compartir lo que sabemos 0 ignoramos... (p. 3)

Hace ya cinco años desde que Aguilera realizara estas afirmaciones y, en este breve lapso temporal, este fenómeno ha ido en frenético aumento. Hoy las Redes Sociales y los Smartphone ${ }^{1}$ se han convertido, prácticamente, en un elemento "obligado" para todo el mundo, cada vez a edades más tempranas.

No es extraño ir por la calle y ver cómo las personas más jóvenes con las que nos a nuestro paso, encontrándose solas o acompañadas, están con su teléfono de la mano, constantemente pendientes de sus pantallas, lejos de interaccionar con el resto de personas que hay a su alrededor, incluso a su lado. De esta forma, en el presente trabajo pretendemos analizar los motivos que hacen tan atractivas estas herramientas y cómo afectan a la vida de los usuarios, centrándonos en diferentes aspectos del comportamiento social de los mismos llegando, de esta forma, a sustituir la comunicación interpersonal cara a cara, directa y presencial por la comunicación a través de estos medios. 
Kram (2016) define las Redes Sociales como plataformas virtuales donde sus usuarios socializan con otras personas desde cualquier dispositivo que posea conexión a internet. Estas redes virtuales, permiten a las personas crear un "perfil", donde colgar información que los caracterice y a la cual otros usuarios de la misma red tienen acceso.

Por su parte, Echeburúa (2012), define las Redes Sociales como "espacios virtuales creados para las relaciones interpersonales, pero habitualmente operan como medios para articular relaciones reales, 0 al menos se orientan con esa expectativa" (p. 22).

Y en la actualidad es realmente así, especialmente entre la población más joven: muchas nuevas relaciones se crean a través de las redes, así como otras tantas se mantienen por esta vía. El problema puede surgir cuando este método se convierte en exclusivo y llega a sustituir la comunicación interpersonal.

En un trabajo previo, Echeburúa (2010), señala:

Las Redes Sociales son el espantajo que aleja el fantasma de la exclusión: se vuelcan las emociones, con la protección que ofrece la pantalla, y se comparte el tiempo libre. Uno puede creerse popular porque tiene listas de amigos en las Redes Sociales (p. 92).

Según el mismo autor, las motivaciones para hacer uso de las Redes Sociales son muchas, entre las que están: ser visible ante los demás, reafirmar la identidad ante el grupo 0 estar conectado con los amigos. De tal forma que el anonimato produce terror.

Es decir, de acuerdo con Echeburúa (2012), las personas utilizan tanto las Redes Sociales porque realizan una importante función, que es la relacional, sirviendo para la diversión y el ocio. Y siendo los jóvenes los usuarios más comunes de estas herramientas constituyen, además, la mayor población de riesgo ante las mismas, considerándose la adolescencia un periodo especialmente vulnerable.

En los chats, las relaciones son más fluidas y crean menos ansiedad que en la vida real porque no hay que enfrentarte a la otra persona cara a cara. Pero esto, a su vez, acarrea un deterioro en la capacidad de interacción con los demás, dificultando el desarrollo de habilidades importantes para la socialización real con otras personas.

Una de las razones que esgrime Echeburúa (2012) por la que son tan utilizadas las Redes Sociales es la opción de publicar y compartir fotos con los demás, lo que supone una actividad de socialización, contribuyendo a la formación de la identidad; es decir, al proceso de forjar el autoconcepto y fortalecer la autoestima.

En artículo del Centro de Adicciones de Barcelona Can Roselló (s.f.) se afirma que algunos psicólogos advierten que exhibir constantemente la vida privada puede ser síntoma de una baja autoestima, buscando la aprobación y aceptación de los demás. Además, se sostiene que, según algunos sociólogos, en las Redes Sociales las personas muestran lo que se quieren mostrar, construyendo una realidad con el fin de recibir retroalimentación. Se plantea así la cuestión de si la egolatría es una reacción inevitable al uso de las Redes Sociales. Según el mismo trabajo, publicar aspectos de la vida personal en las redes se debe a motivos que van desde la diversión o el gusto por compartir momentos con los amigos, hasta presumir de logros, mostrar la propia belleza a los demás 0 , incluso, enviar un mensaje indirecto a alguien.

De acuerdo con esto, Vicens (2016) explica en su artículo "Selfie ${ }^{2}$ de un narcisista", cómo la demostración de lo maravillosa que es nuestra vida a través de la actualización constante de los estados en las Redes Sociales, así como la subida continua de "selfies" en una sociedad donde impera el culto a la imagen son, muchas veces, indicadores de una baja autoestima. Apunta cómo estas personas buscan, generalmente, notoriedad y estas prácticas les ayudan a hacerse visibles exhibiendo, además, sus éxitos y utilizando sobremanera el "Yo". Esta vanidad, junto al deseo de ser elogiados, reconocidos y admirados puede convertir a estas personas en auténticos narcisistas. 


\section{RELACIÓN DE LOS MENORES CON LAS REDES SOCIALES Y EL WHATSAPP: CAUSAS Y CONSECUENCIAS}

Detrás del narcisismo hay una personalidad muy frágil, insegura, que tiene una autoestima muy baja y que necesita ir reafirmándose constantemente desde el exterior. Confunden el yo-ideal y el yoreal. Y además sufren mucho cuando el contador de likes no sube (Vicens, 2016, s.p.)

De esta forma, la acción de darle al botón de like ${ }^{3}$, puede acabar convirtiéndose en una forma de reconocimiento social y terapia particular para sentirse bien con uno mismo. Sin embargo, la necesidad de retroalimentación puede llegar a suponer la pérdida de contacto con la realidad, limitando la vida exclusivamente a la red, donde se puede tener y mostrar una "vida ideal" que en la realidad no se tiene.

Por último, otra de las consecuencias que puede tener un uso indebido de las Redes Sociales y el WhatsApp, es la posibilidad de control que otorgan, ya que permiten estar en contacto continuo, incluso conocer dónde y cuándo la otra persona se ha conectado, afectando de este modo a las relaciones interpersonales.

Elena escribió ayer, por última vez, a las cuatro y media de la madrugada y hoy aún no ha dado señales de vida; Tania está ahora mismo en línea; Marcelino ha madrugado, a las seis y media de la mañana ya estaba conectado. Es información obtenida a través de un teléfono móvil y para conseguirla basta con clicar sobre cada uno de los contactos del WhatsApp (Ricou, 2012)

\section{OBJETIVOS DE LA INVESTIGACIÓN}

Objetivos generales:

Comprobar la relación que establecen los menores con las Redes Sociales y el WhatsApp.

Detectar posibles problemas y consecuencias negativas en la vida de los menores derivados del uso de estas herramientas.

Objetivos específicos:

Conocer la frecuencia y finalidad con la que se utilizan las Redes Sociales.

Comprobar el nivel de confusión entre la vida real y lo mostrado en las Redes Sociales.

Relacionar el posible uso de las Redes Sociales y el WhatsApp con un deterioro de la comunicación interpersonal y la capacidad de resolución de conflictos.

Relacionar la preocupación por colgar fotografías e información personal en las Redes Sociales con carencias en la vida personal, como la falta de autoestima.

Conocer las posibles causas, especialmente sociales, que pueden llevar a una persona a aislarse a través del uso de las Redes Sociales.

\section{METODOLOGÍA}

Nuestra investigación ha sido llevada a cabo a través de un cuestionario ad hoc, de elaboración propia aplicado a una muestra final constituida por $n=134$ sujetos, todos ellos menores de edad, estudiantes de la provincia de Salamanca, participando alumnos de $1^{0}$ a $4^{0}$ de la Enseñanza Secundaria Obligatoria (ESO), 1ํy y 0 de Bachillerato y Formación Profesional Básica (FP), a los cuales se pasaron los cuestionarios de forma escrita. Una vez rellenados todos los formularios, incluimos los datos en el programa de análisis estadístico SPSS, a través del cual se procesaron.

En la Figura 1 y la Figura 2, podemos ver la distribución de la muestra por sexos (53,68\% mujeres y $46,32 \%$ hombres) y repartida según los diferentes cursos en los que se encontraban los menores encuestados, habiendo mayor representación de alumnos de $3^{\circ}$ de la ESO. De forma que hemos obtenido una muestra bastante equitativa en lo que al género se refiere, con una edad media de 14,8 años. 
Figura 1. Distribución de la muestra por sexo

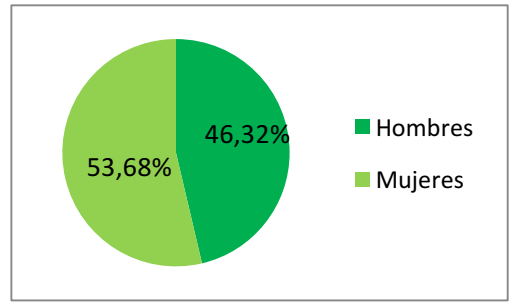

Figura 2. Distribución de la muestra por curso

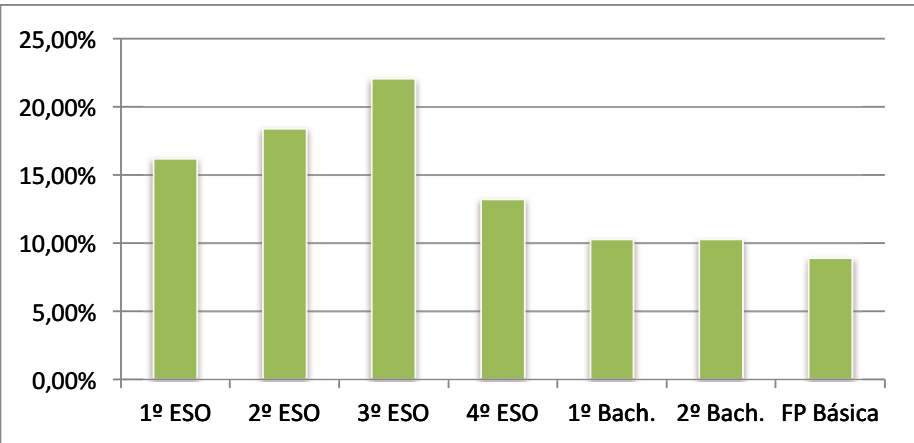

\section{RESULTADOS Y DISCUSIÓN}

Como ya señalábamos en los antecedentes, el WhatsApp es una aplicación prácticamente obligada en los móviles modernos y, de acuerdo con esto, un abrumador $100 \%$ de los estudiantes han indicado que, efectivamente, utilizan esta herramienta de comunicación.

En cuanto al tema concreto de las Redes Sociales, un alto porcentaje de la muestra, el 88,91\%, han admitido tener uno 0 varios perfiles en las mismas. Además, el 82,4\% indican tener instalada alguna de estas redes en sus móviles, recibiendo y atendiendo las notificaciones al instante. Lo que supone, realmente, estar siempre conectado.

Respecto a la frecuencia con la que utilizan dichas redes, el 69,8\% reconoce utilizarlas todos los días más de una hora diaria. Dato directamente relacionado con el porcentaje que asegura tenerlas instaladas en sus móviles. Lo cual significa es que durante más de una hora diaria todos los días estas personas están dedicando un tiempo amplio a estar conectados, tiempo que, obviamente, antes de tal uso dedicarían a otras cosas, probablemente más productivas 0 , simplemente, a relacionarse con sus amigos de manera directa y no a través de una pantalla. Se podría decir que las Redes Sociales están marcando la vida de estos menores, delimitando sus actividades y ocupando cada vez un mayor tiempo de las mismas.

En relación a los motivos del uso de las Redes Sociales, los más representativos son: "para estar en contacto con mis amigos" y "para estar informado de lo que pasa a mi alrededor".

Respecto a la primera afirmación, cabe destacar cómo se contrapone a los resultados hallados en una investigación "antigua" realizada por Muñoz-Rivas, Navarro y Ortegade (2003), en la cual "estar en contacto con los amigos" suponía la tercera razón del uso de Internet, con solo un 11,5\% 


\section{RELACIÓN DE LOS MENORES CON LAS REDES SOCIALES Y EL WHATSAPP: CAUSAS Y CONSECUENCIAS}

de respuestas. Nosotros encontramos que, actualmente, la comunicación se ha convertido en un motivo básico -aglutinando alrededor de un $80 \%$ de la muestra que señala este ítem como una de las motivaciones fundamentales-.

La siguiente afirmación más apuntada es "para saber en todo momento de mis amigos y conocidos", indicando cómo las Redes Sociales se han convertido en una manera no solo de relacionarse, sino de estar permanentemente al tanto de la vida de los demás. En este sentido, no es de desdeñar el potencial riego que entraña este motivo, pudiendo suponer una forma importante de control hacia los otros y una posible expansión de estereotipos y prejuicios guiados, simplemente, por lo que una persona "cuelga" en sus redes, que no es más que una simplificación artificiosa de lo que esa persona quiere mostrar.

Por su parte, aunque los sujetos de la muestra admiten utilizar diferentes Redes Sociales, Facebook sigue siendo una red muy popular entre los jóvenes, por lo que hemos querido obtener una serie de datos referidos específicamente a la misma.

Figura 3a. Representación de no de amigos en Facebook

Figura 3b. Relación 'real' respecto a la Figura 3a
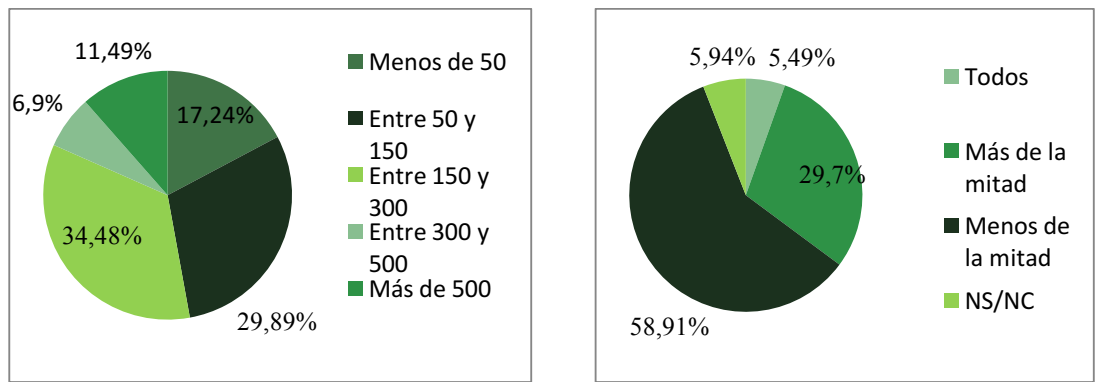

En las Figuras 3a y 3b podemos observar, en primer lugar, el número de amigos que tienen en su Facebook nuestros encuestados y, por su parte, la relación real que afirman tener con dichas personas. De esta forma, podemos ver cómo predomina el rango de entre 150 y 300 amigos. Por su parte, Ilama la atención cierta falta de término medio, ya que la franja de entre 300 y 500 amigos no llega a un $7 \%$ y, sin embargo, casi un $12 \%$ afirma tener más de 500 amigos.

Pero lo más interesante no es tanto el número de amigos que cada cual tenga, sino la relación real mantenida con esas personas porque ¿es realmente posible tener más de 500 amigos y mantener una relación real con tanta gente 0 , por el contrario, es mera apariencia? Cómo respuesta a esta pregunta, en la Figura 3b podemos comprobar cómo una importante mayoría reconoce tener relación con menos de la mitad de las personas que tiene agregadas como "amigos".

Respecto al grado en que se utilizan las Redes Sociales para colgar información y, sobre todo, fotos personales, podemos comprobar en la Figura 4, cómo la mayoría $(66,39 \%)$ de los sujetos de la muestra han colgado alguna vez imágenes suyas en las Redes Sociales. Por su parte, el 20,49\% afirma que sube con frecuencia fotos suyas, siendo un escueto $4,92 \%$ de la muestra que señala subir fotos en las que salen con otras personas. Así, podemos asegurar que predomina la moda de los selfies.

Por el contrario, solo el $8,2 \%$ de la muestra afirma no haber mostrado nunca una imagen suya en las redes, lo que podría corresponderse con el porcentaje de sujetos que se dedica a mirar los tablones de los demás, sin exponerse ellos mismos. 
Figura 6. Muestra de la frecuencia con la que se comprueba quien ha dado like en sus publicaciones

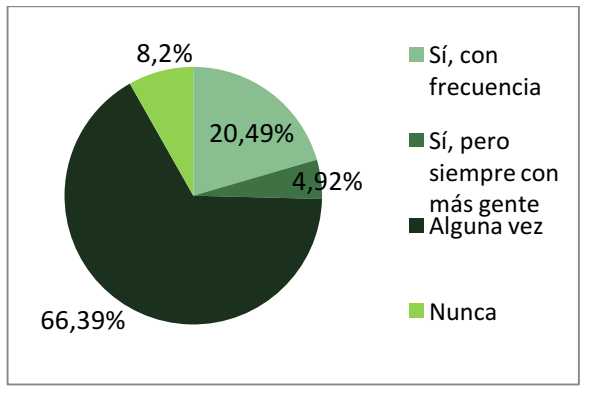

En relación concreta a la cuestión de los selfies, como podemos deducir de la Figura 5, se trata de una moda que ha calado bastante en los jóvenes, siento un $32,79 \%$ de estudiantes los que afirman subir este tipo de fotos individuales con frecuencia. De ellos, solo un $19,57 \%$ dice tener una explicación para dicha conducta, el resto reconocen no tener un motivo para hacerlo, de forma que lo hacen de manera automática, sin pensar sobre ello.

Figura 5. Porcentaje de sujetos que suben con frecuencia selfies individuales a las Redes Sociales

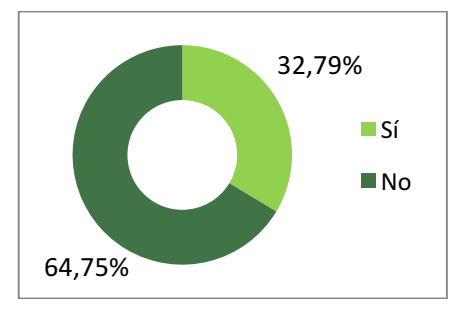

Por su parte, como ya se ha señalado, el reconocimiento a través de likes que se obtiene de las publicaciones y fotos colgadas supone una especie de retroalimentación.

De este modo, se preguntó a los encuestados si consideran que el número de likes que obtienen sus fotos pueden mejorar o empeorar su estado de ánimo a lo que, de manera general, se respondió que no, pero un importante 15,24\%, reconoce que sí le afecta. Esto implica una necesidad de reconocimiento externo importante, pudiendo afectar considerablemente a la vida de la persona.

Continuando con la cuestión de los likes, aunque solo una minoría reconoce abiertamente que el número de los mismos afecta a su estado de ánimo, quizá no sea la cantidad sino quién los emite; relacionando directamente un like con una actitud o un sentimiento personal, cómo el hecho de demostrar interés por alguien de esta forma.

En este caso, como podemos apreciar en la Figura 6, la mayoría de los sujetos (83,57\%) reconocen que alguna vez han comprobado quién ha pulsado ese "botón" en sus publicaciones. Por su parte, un importante 19,01\%, admite hacer esta comprobación siempre, lo que podría llegar a convertirse en una obsesión por saber qué reconocimiento o qué interés tienen los demás hacia uno, corriendo uno de los riesgos mencionados al principio como es llegar a confundir lo que sucede en las redes con la realidad. 


\section{RELACIÓN DE LOS MENORES CON LAS REDES SOCIALES Y EL WHATSAPP: CAUSAS Y CONSECUENCIAS}

Figura 6. Muestra de la frecuencia con la que se comprueba quien ha dado like en sus publicaciones

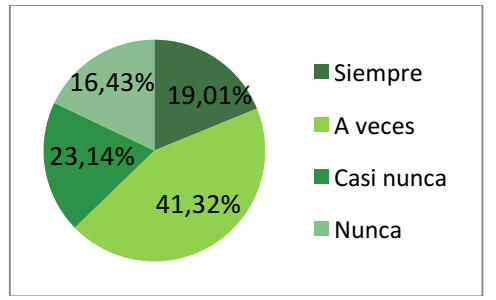

Por su parte, planteamos la cuestión de en qué grado cuelgan aspectos de su vida privada en general -no solo fotos, sino información personal de lo que hacen, dónde están, etc.- (ver Figura 7). La mayoría, un 61,79\%, contesta que "Poco", de lo que se extrae que dentro de este resultado viene implícito que en algunos momentos estas personas han expuesto su vida personal en la red. Por su parte, solo un cuarto de la muestra $(25,2 \%)$ asegura no haber mostrado nunca su vida personal en las redes. En el otro extremo, hay un exiguo 1,63\% de sujetos que asumen exhibir mucho su vida personal, frente a un superior $11,38 \%$ que reconoce hacerlo bastante. Ante estos datos cabe preguntarse ¿dónde trazan el límite entre hacerlo mucho, bastante o poco? Al delimitarlo ellos mismos, es lógico que predominen los valores más bajos pues, de alguna manera, sirve de autoconvicción para pensar que no se están excediendo.

Figura 7. Medida en que publican aspectos de su vida personal en las Redes Sociales

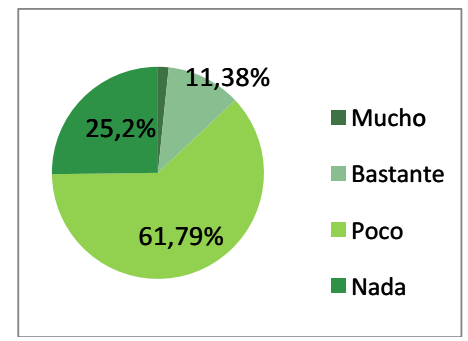

Junto con esta cuestión, planteamos una serie de ítems para saber cuáles son los motivos principales por los que publican aspectos de su vida personal en las redes, siendo la razón más marcada la de compartir momentos con sus amigos. De nuevo otro ejemplo de cómo este tipo de comunicación sustituye a la comunicación interpersonal, donde, por ejemplo, en vez de quedar con los amigos y contarse lo que han hecho el fin de semana se pueden limitar a colgar las fotos y ahorrarse ese "trámite".

En relación a esto, la mayoría de los encuestados (73,1\%) asegura no haberse preocupado nunca en caso de que una publicación suya no haya tenido la repercusión esperada, seguido de un $23,58 \%$ que reconocen que alguna vez sí les ha influido; existiendo un $2,44 \%$ de sujetos que afirma que siempre les preocupa la repercusión de sus publicaciones.

Por último, queríamos comprobar si el uso de esas herramientas afecta a la capacidad de resolución de conflictos de los menores y a su forma de comunicación. Así, en la Figura 8 podemos observar cómo son pocos (7,32\%) los que admiten resolver sus conflictos por WhatsApp en un pri- 
mer momento. Por el contrario, el 26,02\%, afirma solucionar el problema cara a cara con la otra persona. Por su parte, el $27,64 \%$ de los sujetos acepta que en un primer momento lo habla por WhatsApp y valora la situación y posteriormente, si lo consideran, lo habla en persona. Es decir, que la decisión la toman por una interacción en la que no están viendo a la otra persona. Por último, el $39,02 \%$ de los individuos de la muestra afirma que dependiendo de la situación concreta de que se trate actúan de una forma u otra. A la luz de estos resultados, los sujetos parecen potenciar la comunicación indirecta, vía WhatsApp, frente a la comunicación personal presencial y consideran a la primera una forma válida de afrontamiento de conflictos aunque, manifiestamente, implique poner en práctica menos habilidades sociales y de comunicación.

Figura 8. Forma de resolución de conflictos

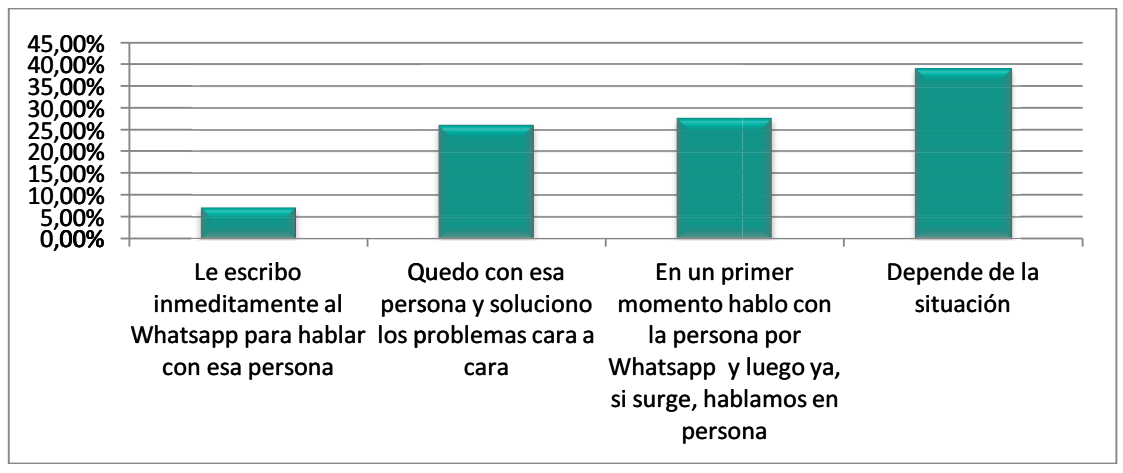

\section{CONCLUSIONES}

Según los resultados obtenidos, podemos afirmar que el uso de las Redes Sociales y el WhatsApp es bastante elevado, así como también es muy alta la frecuencia general con la que se utilizan; de forma que se han convertido en una parte importante de la vida de los menores, lo que a su vez tiene importantes efectos en diferentes aspectos de la vida social de los mismos.

El hecho de comunicarse a través de una pantalla se ha convertido, en muchas ocasiones, en un sustituto de la comunicación interpersonal, lo que supone la pérdida progresiva de habilidades sociales y de comunicación. Se está llegando a extremos en los que la pantalla de un móvil o un ordenador están cobrando más importancia que el contacto real con los demás, lo que supone una triste y peligrosa realidad.

Además de la comunicación a través de mensajería instantánea, la interacción se puede llevar a cabo a través de la subida de fotos, videos, información, comentarios, etc., una práctica que también puede llegar a suponer un riesgo cuando se llega a confundir lo que se muestra en las redes con la realidad. De esta forma, hay personas que exhiben sus vidas, logros o fotografías -en las que salen especialmente bien- con el objetivo de recibir retroalimentación a través de likes, como si se tratase de un reconocimiento. Son particularmente vulnerables a estas acciones aquellas personas con baja autoestima o carencias en su vida personal, cuyo estado de ánimo puede mejorar o empeorar en función de la retroalimentación obtenida en las redes.

Como conclusión final, queremos resaltar y resumir los riesgos que puede tener un mal uso de las Redes Sociales y aplicaciones como WhatsApp, entre los que encontramos un deterioro de las habilidades sociales y de comunicación, dificultando la capacidad de relación y socialización con otras personas y, por consiguiente, aislando, mermando y delimitando considerablemente la vida de 
la persona. Además de esto, puede incluir otros riesgos como el control, la presión por cuestiones como el culto a la imagen o la confusión de la realidad con el mundo virtual, entre otros.

\section{REFERENCIAS BIBLIOGRÁFICAS}

Aguilera, M. (2013). Desde mi teclado. Crítica. Redes sociales ¿Necesidad o Adicción?, 985, Recuperado de:

http://www.revistacritica.com/administrator/components/com_avzrevistas/pdfs/111050168b3aea3e dcfcb42a6cdeaaa1-985-Redes-sociales-necesidad-0-adicci-n.pdf

Centro Can Roselló (s.f.). El síndrome del selfie: De la moda al narcisismo. Recuperado 14 de marzo de 2017, de http://www.centroadiccionesbarcelona.com/el-sindrome-selfie-de-la-moda-al-narcisismo/

Echeburúa, E. (2010). Adicción a las nuevas tecnologías y a las Redes Sociales en jóvenes: Un nuevo reto. Revista Adicciones, 22(2), 91-96.

Echeburúa, E. (2012). Adicción a las redes sociales y nuevas tecnologías en niños y adolescentes (1. ${ }^{\text {a }}$ ed.). Madrid: Ediciones Pirámide.

Kram, C. (2016). Los vínculos de los jóvenes en el contexto de las Redes Sociales. Trabajo de Fin de Grado. Universidad Nacional de San Luis, Argentina.

Muñoz-Rivas, M. J., Navarro, M. E. y Ortegade Pablo, N. (2003). Patrones de uso de Internet en población universitaria española. Adicciones, 15(2), 137-144.

Ricou, J. (2012, noviembre 4). Cómo afecta el Whatsapp a las relaciones de pareja. La Vanguardia. Recuperado 13 de abril 2017, de:

http://www.lavanguardia.com/vida/20121104/54354818574/whatsapp-relaciones-pareja.html

Vicens, S. (2016). Selfie de un narcisista. Independientes, Revista Especializada en Adicciones. Recuperado de: http://revistaindependientes.com/selfie-de-un-narcisista

1 Teléfono inteligente. Hace referencia a aquellos móviles que pueden realizar funciones semejantes a las de un ordenador.

2 Autofoto 0 autoretrato.

3 "Me gusta" que le podemos dar la publicación o foto de una persona en algunas Redes Sociales, como manera de reconocimiento o muestra de agrado ante la misma. 